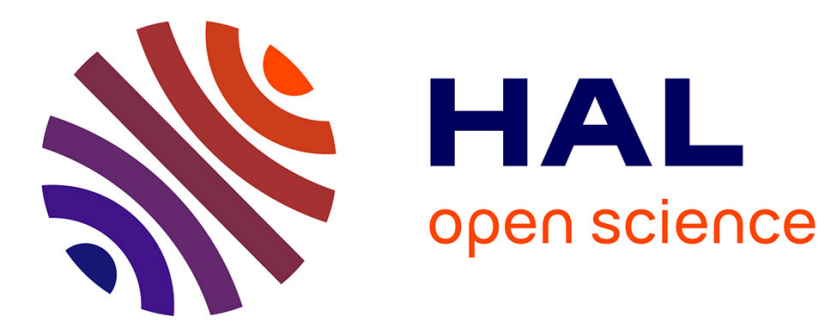

\title{
Robust Fringe Detection Based on Bi-Wavelet Transform for Self-Mixing Displacement Sensor
}

Olivier Bernal, Han Cheng Seat, Usman Zabit, Frédéric Surre, Thierry Bosch

\section{To cite this version:}

Olivier Bernal, Han Cheng Seat, Usman Zabit, Frédéric Surre, Thierry Bosch. Robust Fringe Detection Based on Bi-Wavelet Transform for Self-Mixing Displacement Sensor. SENSORS, 2015 IEEE , Nov 2015, Busan, South Korea. 10.1109/ICSENS.2015.7370375 . hal-01262273

\section{HAL Id: hal-01262273 \\ https://hal.science/hal-01262273}

Submitted on 15 Feb 2016

HAL is a multi-disciplinary open access archive for the deposit and dissemination of scientific research documents, whether they are published or not. The documents may come from teaching and research institutions in France or abroad, or from public or private research centers.
L'archive ouverte pluridisciplinaire HAL, est destinée au dépôt et à la diffusion de documents scientifiques de niveau recherche, publiés ou non, émanant des établissements d'enseignement et de recherche français ou étrangers, des laboratoires publics ou privés. 


\title{
Robust Fringe Detection Based on Bi-Wavelet Transform for Self-Mixing Displacement Sensor
}

\author{
O. D. Bernal, H.C. Seat, U. Zabit, F. Surre and T. Bosch \\ doi: http://dx.doi.org/10.1109/ICSENS . 2015.7370375
}

\begin{abstract}
A novel signal processing method based on custom-made Wavelet Transform (WT) is presented for robust detection of fringes contained in the interferometric signal of Self-Mixing (SM) laser diode sensors. It enables the measurement of arbitrarily-shaped vibrations even in the corruptive presence of speckle. Our algorithm is based on the pattern recognition capability of the customized WT for identifying SM fringes. Once the fringes have been correctly detected, phase unwrapping methods can be applied to retrieve the complete instantaneous phase of the SM signals. Here, the novelty consists in using two distinct mother wavelets $\Psi_{r}(\mathrm{t})$ and $\Psi_{d}(\mathrm{t})$ particularly designed to distinguish SM patterns as well as the displacement direction. The peaks, i.e. maxima of WT, then allow the detection of the fringes.
\end{abstract}

\section{Introduction}

Self-mixing (SM) or optical feedback interferometry has been widely used for metrological sensing applications during the last two decades as it results in a simple, compact, self-aligned, and cost-effective sensor [8]. SM effect occurs in a laser when a part of the beam backscattered by a target is coupled back into the laser cavity and causes interference with the emitted beam, thus modifying the spectral properties of the laser. The variations in the optical output power of the laser diode $\mathrm{P}(\mathrm{t})$ caused by this optical feedback can be written as [16]:

$$
P(t)=P_{0}\left[1+m \cos \left(x_{F}(t)\right)\right]
$$

where $P_{0}$ is the emitted optical power under free-running conditions, $m$ the modulation index and $x_{F}(t)$ the laser output phase in the presence of feedback, given

doi: http://dx.doi.org/10.1109/ICSENS .2015.7370375 
by:

$$
x_{F}(t)=2 \pi \frac{D(t)}{\lambda_{F}(t) / 2}
$$

with $D(t)$ the target displacement. The emission wavelength subject to feedback $\lambda_{F}(t)$ is intrinsically encompassed in the phase equation [5]:

$$
x_{0}(t)=x_{F}(t)+C \sin \left[x_{F}(t)+\arctan (\alpha)\right]
$$

where $\alpha$ is the linewidth enhancement factor and $x_{0}(t)$ is the laser output phase in the absence of feedback, given by:

$$
x_{0}(t)=2 \pi \frac{D(t)}{\lambda_{0} / 2}
$$

with $\lambda_{0}$ is the emission wavelength under free running conditions. The feedback coupling factor $C$ is then [8]:

$$
C=\frac{\tau_{D}}{\tau_{L}} \gamma \sqrt{1+\alpha^{2}} \kappa_{e x t}
$$

where $\tau_{L}$ and $\tau_{D}$ are the round trip times in the internal and external cavities respectively, $\gamma$ is the coupling efficiency and $\kappa_{e x t}$ depends linearly on the surface reflectivity of the target.

The $C$ parameter plays a very important role in SM signals as variations in $C$ induce changes in the so-called SM operating regimes [8]. Thus, the shape of SM signals strongly depends on $C$. As a result, the $C$ factor [13] is usually used to distinguish between three main optical feedback regimes [9]:

- $0.1<C<1$ : weak optical feedback regime with sinusoidal or asymmetric SMI fringes devoid of sharp discontinuities [7].

- $1<C<4.6$ : moderate optical feedback regime with sawtooth-like SMI fringes exhibiting hysteresis [3].

- $4.6<C$ : strong optical feedback regime [9, 14].

Consequently, for practical applications, a complex variety of SM signals needs to be reliably processed as variations in optical feedback can become unavoidable. Typically, each SM regime would thus require specific signal processing for displacement extraction [16]. This is further aggravated if speckle is additionally present.

In this context, a robust SM fringe detection scheme (ideally independent of SM feedback regime, additive white noise, and speckle effect causing amplitude modulation of signal) is necessary in order to perform the target displacement

doi: http://dx.doi.org/10.1109/ICSENS.2015.7370375 
reconstruction because most of the signal processing techniques rely on it as a first step [4, 5]. Usually, the moderate optical feedback regime is thus sought for displacement sensing as its supposedly straight-forward signal shape [5] leads to simplified signal processing. However such a regime cannot always be obtained. Consequently, different methods have been proposed to improve the robustness of SM fringe detection, as detailed below.

Signal processing based on envelope tracking and adaptive threshold scheme [15] was shown to improve the robustness of displacement reconstruction but in some cases, fringes might not be detected. A Hilbert transform-based algorithm [1] was then proposed to extract the instantaneous SM phase. Nevertheless, it cannot inherently determine the target direction and sub-wavelength displacements could be wrongly interpreted. WT [6] was shown to be an efficient way to analyze SM signals as non-stationary signals. However, such approaches were hitherto based on available mother wavelets. Consequently, differential and evolutionary algorithms [10] were necessary to detect the fringes, but these require high computing resources.

In this paper, it is shown that the robustness of fringe detection can be enhanced by the use of dedicated mother wavelets specifically defined for SM signals in order to greatly alleviate the computing demand. The following sections describe the proposed signal processing and the results obtained for simulated and experimentally acquired SM signals which demonstrate an amelioration in the final displacement sensing results.

\section{Fringe Detection using a Bi-Wavelet Transform}

\subsection{Wavelet transform and non stationary signals}

Contrarily to Fourier Transform, WT can provide information of spectrum changes with respect to time [12]. As a result, WTs are better suited to analyze nonstationary signals. The WT decomposes the signal into different scales with different levels of resolution by dilating a single function named the mother wavelet $\Psi$. The definition of the wavelet transform of a signal $f(t)$ is as follows:

$$
W f(s, t)=f(t) * \Psi_{s}=\int f(u) \Psi\left(\frac{t-u}{s}\right) d u
$$

where $\Psi$ is the mother wavelet and s the scale parameter which provides information regarding the signal frequency.

For the detection of singularities with such multiscale transforms, the technique based on the modulus maxima can be used [12]. Here, the singularities to be detected are the SM fringes, each of which corresponds to $\lambda / 2$ target displacement.

doi: http://dx.doi.org/10.1109/ICSENS.2015.7370375 


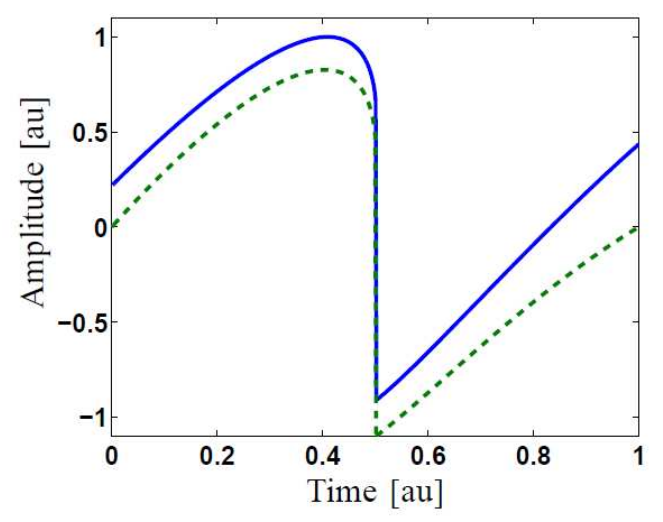

Figure 1: Original Pattern based on $\mathrm{SM}$ rising fringe obtained for $\mathrm{C}=1.5$ (blue line) and its Adapted Wavelet $\Psi_{r}$ (dashed green line)

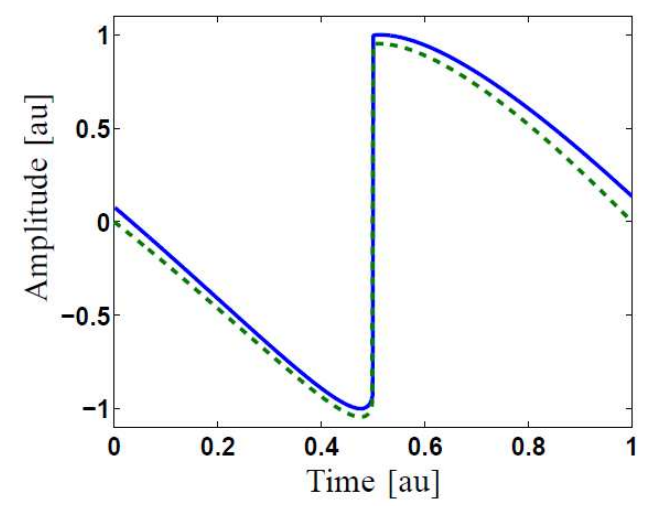

Figure 2: Original Pattern based on SM decreasing fringe obtained for $\mathrm{C}=1.5$ (blue line) and its Adapted Wavelet $\Psi_{d}$ (dashed green line)

\subsection{The proposed Bi-wavelet tranform approach}

Different kinds of mother wavelets can be used to analyze SM signals such as the symlet wavelets and the Daubechies wavelets. However, different results are obtained depending on the mother wavelet used [6]. Hence, in order to obtain more accurate information about the presence or absence of a SM fringe, we have created specific mother wavelets tailor-made for SM signals. Noting that the WT is based on convolution (eq.(6)), WT can thus also be used as a pattern recognition method. Further, WT inherently computes the cross-correlation along the scales.

Consequently, if the mother wavelet is defined to ressemble an SM fringe, it can then give better results as it will address more specific singularities. In

doi: http://dx.doi.org/10.1109/ICSENS.2015.7370375 
addition, as SM signal fringes for moderate and weak regimes are not symmetric [2], it can be advantageous and more efficient to define not just one but two mother wavelets to provide a better sensitivity to fringe detection and also to discriminate the displacement direction in an intrinsic manner.

Here, using the Matlab $($ wavelet toolbox, based on a typical rising and decreasing SM fringe for $C=1.5$, two mother wavelets $\Psi_{r}(t)$ and $\Psi_{d}(t)$ are thus defined respectively (Fig. 1 and 2). This $C$ value has been chosen to match our SM sensors autofocus system which tries to lock to $C \approx 1.5$ [4]. The generated function $\Psi_{r}$ and $\Psi_{d}$ can be considered to be wavelets as:

$$
\begin{gathered}
\int_{-\infty}^{\infty} \Psi_{r, d}(t) d t=0 \\
\int_{-\infty}^{\infty}\left|\Psi_{r, d}\right|^{2}(t) d t<\infty
\end{gathered}
$$

As in [11], the maxima are selected by using a threshold based on the signalto-noise ratio of the signal modulus. Finally, a tracking envelope of those maxima obtained for each mother wavelet is used to distinguish the maxima into corresponding rising or decreasing fringe patterns.

\section{Simulation Results}

Initially, a simulated noisy sinusoidal target displacement is retrieved using the biWT approach for a low $C$ value $(C \approx 0.6)$ to demonstrate that the algorithm is not just restricted to the moderate feedback regime. Fig. 3 clearly shows that $\Psi_{r}(t)$ and $\Psi_{d}(t)$ are particularly adapted to rising and decreasing fringes respectively. Thus, SM fringes have been correctly detected in spite of presence of noise and variable fringe amplitude.

Further, Fig 4 shows the WT of the same SM signal (as in Fig. 3 c)) using only one standard reverse biorthogonal mother wavelet $\Psi_{\text {rbio }}$. Compared with the proposed approach, it is much more complicated to discriminate the relevant singularities using the modulus maxima approach. For instance, if the red dashed line in Fig. 4 is defined as the threshold, then 6 false rising-fringes are detected. On the contrary, if the green dashed line in Fig. 4 is used as the threshold, then one decreasing-fringe is not detected.

The robustness of the proposed approach regarding speckle is next tested in Fig. 4 showing a simulated SM signal with a modulated amplitude (factor of 8) as well as a modulated $C$ value within 1-3. It demonstrates that our proposed method can reconstruct the target displacement even in the presence of speckle. 

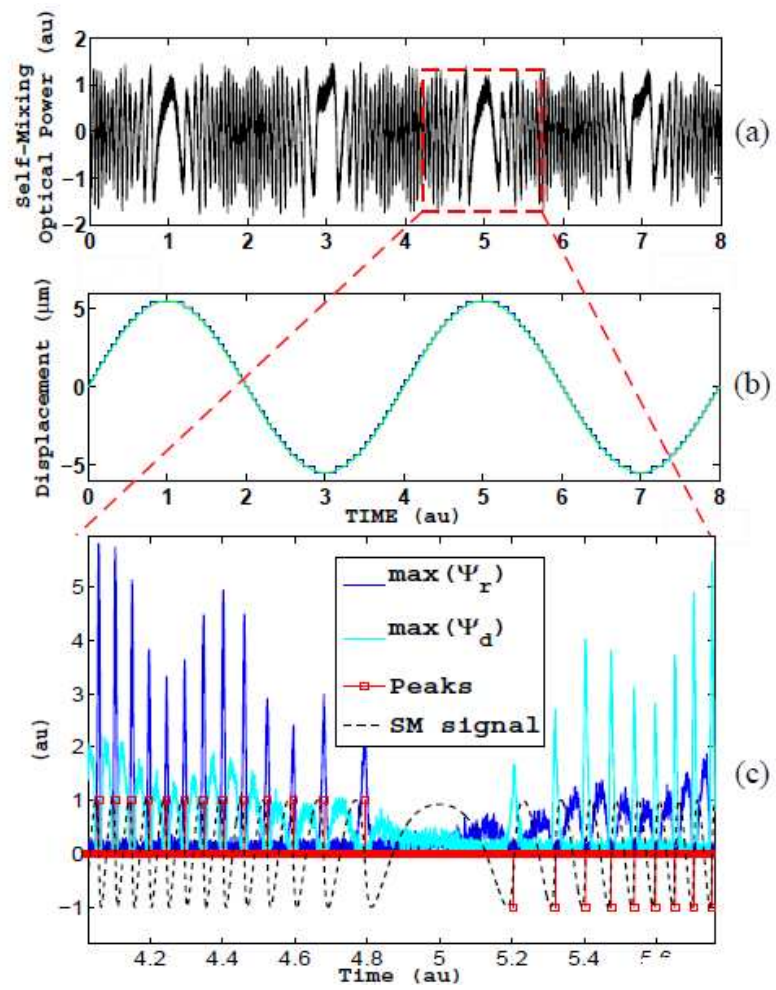

Figure 3: (a) Simulated noisy modulated SM signal (black line) with $\mathrm{C}=0.6$. (b) Reconstructed displacement based on fringe counting (blue line) using bi-WT compared to the original displacement (green line). (c) Zoom showing the fringe detection mechanism based on $\Psi_{r}(\mathrm{t})$ and $\Psi_{d}(\mathrm{t})$. Black dashed line: SM signal (without noise and modulation) added for clarity of comparison

\section{Experimental Results}

The proposed fringe detection method has been experimentally tested. The LD used in the SM sensor, driven by a constant injection current of $30 \mathrm{~mA}$ and a maximum output power of $50 \mathrm{~mW}$, is a Hitachi HL7851G emitting at $\lambda=785.86 \mathrm{~nm}$. A LT110-P collimating lens with a focal length of $6.24 \mathrm{~mm}$ is employed to collimate the laser beam onto the target. The variations in the optical output power of the LD P(t) are monitored through the built-in photodiode contained in the LD package.

Fig. 6 (a) illustrates the SM signal acquired for a randomly-vibrating target from which the reconstructed displacement of Fig. 6(b) is successfully retrieved via bi-WT, based on fringe counting. Fig. 6 (c) finally highlights the fringe detection results for each wavelet. 


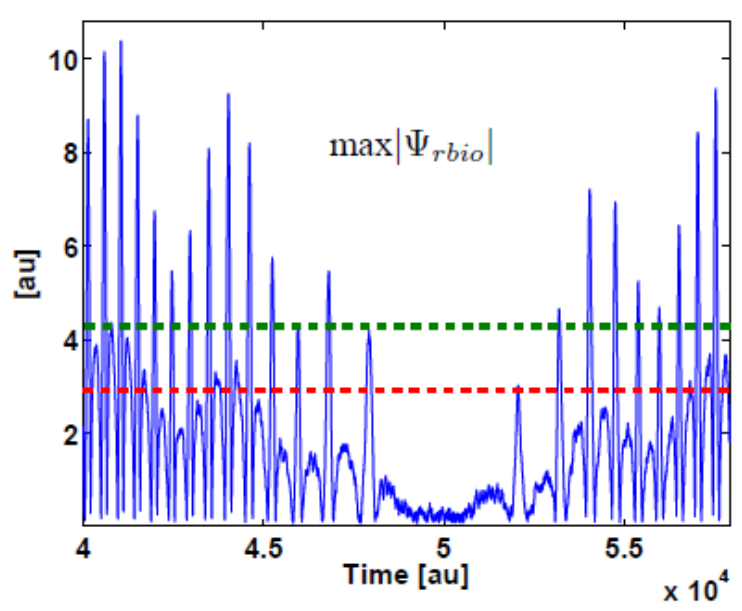

Figure 4: Zoom showing the fringe detection mechanism based on a reverse biorthogonal mother wavelet $\Psi_{r b i o}$ and the modulus maxima method applied on the SM signal of Fig. 3, In red and green dashed lines, two possible threshold positions introducing fringe detection errors.

\section{Conclusion}

In this paper, we have presented a new approach based on the WT technique to perform robust fringe detection for displacement measurements based on optical feedback interferometry for laser subject to weak and moderate feedback regimes. It was also shown that such a method can inherently discriminate the displacement direction. It was successfully tested on different SM signals belonging to varying optical feedback regimes, in the presence of noise and speckle.

The proposed approach is demonstrated to efficiently detect SM signal fringes. However, prior to implementation in a real-time system, operating parameter such as determining the optimum number of scales required still need to be addressed as a function of the system sampling frequency and of the amplitude and frequency range of the moving target.

Furthermore, SM signal denoising based on wavelet maxima can also be performed before applying the proposed approach in order to further enhance its robustness. 


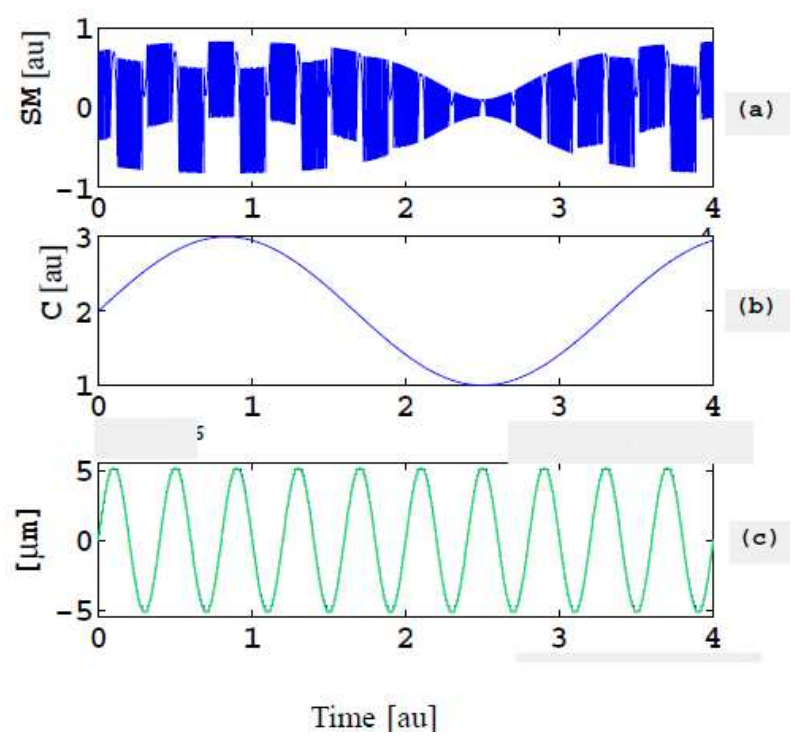

Figure 5: (a) Simulated SM signal affected by speckle but without noise with (b) C variation ranging from 1 to 3 , (c) retrieved displacement after fringe detection and counting (dotted blue line) compared with reference target displacement (green line).

\section{Acknowledgment}

The authors would like to thank F. Jayat for the technical support provided during experimental set-up and electronic circuit board design.

\section{References}

[1] A.L. Arriaga, F. Bony, and T. Bosch. Speckle-insensitive fringe detection method based on hilbert transform for self-mixing interferometry. Applied optics, 53(30):6954-6962, 2014.

[2] O. D Bernal, U. Zabit, and T. Bosch. Classification of laser self-mixing interferometric signal under moderate feedback. Applied optics, 53(4):702708, 2014.

[3] Olivier D Bernal, Usman Zabit, and Thierry Bosch. Classification of laser self-mixing interferometric signal under moderate feedback. Applied optics, 53(4):702-708, 2014.

doi: http://dx.doi.org/10.1109/ICSENS . 2015.7370375 

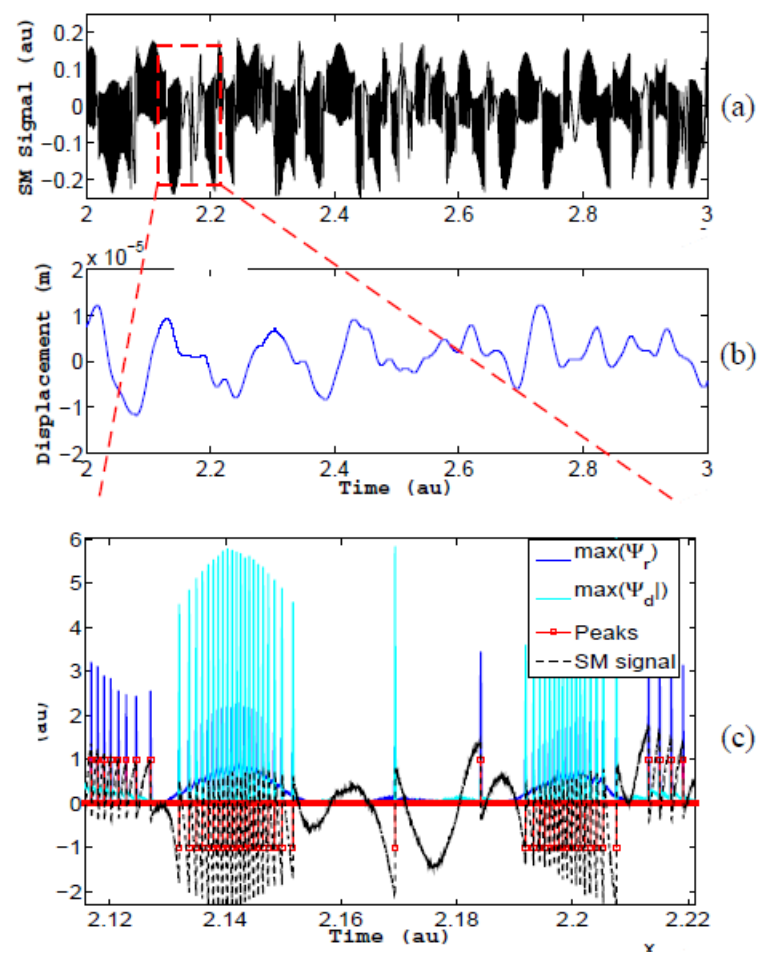

Figure 6: (a) Experimental SM signal from a randomly vibrating target and (b) reconstructed displacement using bi-WT. (c) Zoom of the inset of (a) showing the fringe detection mechanism based on $\Psi_{r}(\mathrm{t})$ and $\Psi_{d}(\mathrm{t})$. Black dashed line represents experimental SM signal for clarity purposes

[4] Olivier Daniel Bernal, Usman Zabit, and Thierry M Bosch. Robust method of stabilization of optical feedback regime by using adaptive optics for a self-mixing micro-interferometer laser displacement sensor. Selected Topics in Quantum Electronics, IEEE Journal of, 21(4):1-8, 2015.

[5] C. Bès, T. Bosch, G. Plantier, and F. Bony. Characterization of a selfmixing displacement sensor under moderate feedback. Optical Engineering, 45(8):084402-084402, 2006.

[6] C. Bès, Guy Plantier, and T. Bosch. Displacement measurements using a self-mixing laser diode under moderate feedback. Instrumentation and Measurement, IEEE Transactions on, 55(4):1101-1105, Aug 2006.

[7] Caroline Bés, Victorien Belloeil, Guy Plantier, Yves Gourinat, and Thierry Bosch. A self-mixing laser sensor design with an extended kalman filter for 
optimal online structural analysis and damping evaluation. Mechatronics, IEEE/ASME Transactions on, 12(3):387-394, 2007.

[8] T. Bosch, C. Bes, L. Scalise, and G. Plantier. Optical feedback interferometry, volume 7, pages 107-127. American Scientific Publishers, 2006.

[9] Silvano Donati. Developing self-mixing interferometry for instrumentation and measurements. Laser \& Photonics Reviews, 6(3):393-417, 2012.

[10] A. Doncescu, C. Bes, and T. Bosch. Displacement estimation with an optical feedback interferometer using an evolutionary algorithm. In Sensors, 2007 IEEE, pages 382-386, Oct 2007.

[11] Taiwen Jiang, Bingzhen Chen, Xiaorong He, and Paul Stuart. Application of steady-state detection method based on wavelet transform. Computers \& chemical engineering, 27(4):569-578, 2003.

[12] S. Mallat and W.L. Hwang. Singularity detection and processing with wavelets. Information Theory, IEEE Transactions on, 38(2):617-643, March 1992.

[13] T. Taimre and A. D. Rakić. On the nature of acket's characteristic parameter c in semiconductor lasers. Applied optics, 53(5):1001-1006, 2014.

[14] Yanguang Yu, Jiangtao Xi, Joe F Chicharo, and Thierry M Bosch. Optical feedback self-mixing interferometry with a large feedback factor: behavior studies. Quantum Electronics, IEEE Journal of, 45(7):840-848, 2009.

[15] U. Zabit, O.D. Bernal, and T. Bosch. Self-mixing laser sensor for large displacements: Signal recovery in the presence of speckle. Sensors Journal, IEEE, 13(2):824-831, Feb 2013.

[16] U. Zabit, T. Bosch, and F. Bony. Adaptive transition detection algorithm for a self-mixing displacement sensor. Sensors Journal, IEEE, 9(12):1879-1886, Dec 2009. 